\title{
Impact of Experimentally Induced Serotonin Deficiency by Tryptophan Depletion on Sleep EEG in Healthy Subjects
}

Ulrich Voderholzer, M.D., Magdolna Hornyak, M.D., Birger Thiel, M.S., Christine Huwig-Poppe, M.D., Andrea Kiemen, M.A., Almut König, M.D., Jutta Backhaus, M.A., Dieter Riemann, Ph.D., Mathias Berger, M.D., and Fritz Hohagen, M.D.

The tryptophan depletion test is a research strategy to investigate the functional consequences of decreasing the brain serotonin metabolism. Because serotonin is involved in sleep regulation and the regulation of affective states, we studied the acute polysomnographic effects of tryptophan depletion and expected to induce similar changes of sleep EEG as observed in depressed patients. A total of 12 healthy subjects (mean age $34 \pm 3$ years) had eight polysomnograms, divided in two blocks of 4 consecutive nights. After one adaptation and 1 baseline night, subjects received a lowprotein diet on day 3 and 4 until midday. On day 4 at $18.00 \mathrm{~h}$, they drank an amino acid mixture either devoid of tryptophan or containing $2.3 \mathrm{~g}$ of tryptophan (placebo control) in randomized and double-blind order, resulting in an $85 \%$ decrease (tryptophan depletion) and a $144 \%$ increase (placebo control) of serum tryptophan at $22.00 \mathrm{~h}$. After tryptophan depletion but not placebo, significant effects on sleep EEG were observed in terms of decreased non-rapid eye movement (non-REM) stage 2, increase of wake \%, and of rapid eye movement (REM) density compared with baseline. REM latency was not altered, however the first and second REM period interval were significantly shorter after tryptophan depletion. This study underlines the impact of the serotonergic system on sleep maintenance and on REM sleep. [Neuropsychopharmacology 18:112-124, 1998] (C) 1998 American College of Neuropsychopharmacology. Published by Elsevier Science Inc.
KEY WORDS: Tryptophan; Sleep; REM sleep; Serotonin; Amino acids; Mood

The tryptophan depletion test was introduced as a research strategy to obtain information on the functional consequences of lowering brain serotonin levels in humans and is based on the finding that serotonin synthesis is dependent on the availability of its precursor tryptophan (Gessa et al. 1974; Fernstrom 1977; Moja et al. 1989). With this test, subjects receive a "drink" con-

From the Department of Psychiatry and Psychotherapy, University Hospital of Freiburg, Freiburg, Germany.

Address correspondence to: Ulrich Voderholzer, M.D., Abteilung für Psychiatrie \& Psychotherapie der Universität Freiburg, Hauptstr. 5, 79104 Freiburg, Germany.

Received February 4, 1997; revised April 14, 1997; accepted April 25,1997 . taining a mixture of amino acids without tryptophan resulting in a $80 \%$ to $90 \%$ decrease of plasma tryptophan levels (Young et al. 1985). Many studies up to now investigated the effect of tryptophan depletion in patients with depressive disorders (Delgado et al. 1990, 1994) to test the hypothesis of abnormal serotonin function in depression (Meltzer and Lowy 1987). Delgado et al. (1990) reported a transient relapse into depression in patients who had a sufficient therapeutic antidepressant response. Also, in patients with seasonal affective disorder being remitted after light therapy, a depressive relapse was induced after tryptophan depletion (Neumeister et al. 1997). On the other hand, in drug-free depressed patients, mood did not further deteriorate after this challenge test (Delgado et al. 1994). Male healthy subjects showed an enhanced susceptibility for a mood- 
lowering effect only if a family history of major affective disorder was present (Benkelfat et al. 1994). By contrast, a lowering of mood was found in euthymic women without a family history of major affective disorder (Ellenbogen et al. 1996). These changes in mood observed in remitted depressive patients and in healthy subjects were interpreted as a consequence of the effects of the tryptophan-deficient amino acid mixture on the central nervous system serotonin metabolism. Recently Cappiello et al. (1996) reported seasonal variation of the prolactin response to L-tryptophan and of baseline tryptophan levels in depressed patients but not in healthy subjects, a result that further supports the hypothesis of an abnormal serotonin function in depression.

Serotonergic receptors are widely distributed in the central nervous system and involved in the regulation of many biological functions such as appetite, neuroendocrine secretion, nociception, sexual behavior, mood, anxiety, aggression, and sleep. Among these functions sleep represents a useful paradigm to investigate the acute response to a manipulation of central serotonergic tone. Its role is integral in both non-rapid eye movement (non-REM) and rapid eye movement (REM) sleep. Whereas the latter is promoted by cholinergic transmission pathways originating in the dorsal tegmentum and inhibited by monoaminergic neurons in the dorsal raphe and nucleus ceruleus, respectively, (Hobson et al. 1975), serotonergic stimulation results in REM sleep inhibition, which was demonstrated after administration of pharmacological stimuli like clomipramine (Kupfer and Ehlers 1989; Riemann and Berger 1990) or fluoxetine (Nicholson and Pascoe 1988). Polysomnographic studies in depression described REM sleep abnormalities such as a decrease in REM latency, an increase in REM percentage and a significantly longer first REM period (for review see Benca et al. 1992; Berger and Riemann 1993). These changes may be interpreted either as consequence of an increased cholinergic tone or a decrease of serotonergic tone. The serotonergic system is also involved in the regulation of non-REM sleep (Jones 1994). The 5-HT precursor tryptophan, which was reported to have hypnotic effects (Hartmann et al. 1974), was widely used as a hypnotic (Schneider-Helmert and Spinweber 1986) until it was withdrawn from the market because of severe side effects such as eosinophilia myalgia syndrome, which was most likely not caused by tryptophan but by soilings during the process of synthesis (Hill et al. 1993; Yamaoka et al. 1994).

It is the hypothesis of this study that an experimentally induced serotonin deficiency by tryptophan depletion might cause a decrease of non-REM sleep and a diminution of REM latency and therefore might be a model for sleep in depression. Bhatti et al. (1995a,b) recently reported a shortening of REM latency and an increase of REM sleep percentage in healthy subjects and in patients with a major depressive disorder in remis- sion being under treatment with serotonergic antidepressants. In both studies, however, no placebo control was performed. Therefore, in this study the effects of tryptophan depletion on sleep were compared with those of a "sham depletion," i.e., an identical test procedure with the only difference that tryptophan was added to the diet and to the amino acid drink.

\section{METHODS}

\section{Subjects}

A total of 13 healthy subjects (age: 23 to 55 years) were recruited for this study through personal contact. One male subject was excluded because of significant sleep disturbance during the baseline night. The remaining subjects had a mean age of $34 \pm 9$ years, six of them were male (mean age: $36 \pm 11$ years) and six female (mean age: $32 \pm 8$ years). The rationale for including male and female subjects in this age range was to obtain data from a sample representative of the adult population, which could serve as a control group for further studies in psychiatric patients. All subjects included were screened by routine laboratory parameters including a urine test, drug screening, ECG, EEG, physical, and neurological examination and were found to be in good physical health. Exclusion criteria were intake of any medication including birth control pills, more than $15 \%$ deviation from the ideal body weight, pregnancy or lactation, own or family history of psychiatric disorders, history of alcohol or drug abuse, insomnia, abnormalities of the gastrointestinal tract, which might interfere with the resorption or metabolism of the amino acid drink, or any other significant medical illness. Written consent was obtained before participation in the study. The experiments were performed in accord with the Helsinki Declaration of 1975. The protocol was approved by the Ethical Committee of the University of Freiburg.

\section{Design}

For the experiments we used a double-blind, randomized cross-over design. All subjects participated in a tryptophan-depletion experimental session and in a placebo control session ("sham depletion"), which was identical apart from the addition of tryptophan to the diet and to the amino acid drink. The study was carried out in the sleep laboratory of the Department for Psychiatry and Psychotherapy of the University of Freiburg. Each session consisted of four days, including 4 sleep polygraphic recordings: (1) adaptation, (2) baseline, (3) diet, and (4) tryptophan depletion or sham depletion. The two sessions were separated by 4 weeks to study the female subjects during the same phase of their menstrual cycle. All female subjects were studied during the 
follicular phase. The first two polysomnograms served for adaptation to the sleep laboratory conditions and to obtain baseline sleep parameters. On day 3 and on day 4 until midday, subjects received standardized nutrition consisting of a low protein diet containing of about $160 \mathrm{mg}$ of tryptophan per day, $18.6 \mathrm{~g}$ of protein and $2,500 \mathrm{kcal}$ for the male subjects and 2,000 kcal for the female subjects. Meals had to be taken in the research ward. In addition to each meal, subjects received one capsule containing either $0.5 \mathrm{~g}$ of L-tryptophan (sham condition) or placebo (tryptophan depletion condition). This diet was similar to the diet used by Delgado et al. (1990). The third night was registered to investigate effects of the low protein diet on sleep (diet night). On day 4 after lunch, subjects were not allowed to take any food or drink except water. At $18.00 \mathrm{~h}$, they received either a tryptophan-free (tryptophan depletion) or a $2.3 \mathrm{~g}$ tryptophan-containing (sham depletion) amino acid drink. The amino acid mixture was the same as used by Young et al. (1985) and other authors (Delgado et al. 1990, 1994). Because of the unpleasant taste, methionine, cysteine, and arginine were given in capsules. Until the beginning of sleep recordings at $23.00 \mathrm{~h}$ and also on the following morning until $10.00 \mathrm{~h}$, subjects did not receive any further food.

\section{Polysomnographic Assessments}

Sleep recordings were made on a 17-channel Nihon Kohden EEG polysomnograph from "lights out" $(23.00 \mathrm{~h})$ to "lights on" $(7.00 \mathrm{~h})$ at a paper speed of $10 \mathrm{~mm} / \mathrm{s}$. All sleep recordings registered EEG (C3-A2; C4-A1), ECG, horizontal EOG, and submental EMG. During the adaptation night, all subjects were screened for apneas and periodic leg movements by monitoring abdominal and thoracic effort, nasal airflow, oxymetry, and bilateral tibialis anterior EMG. More than five apneas or hypopneas per hour or more than five periodic leg movements per hour were exclusion criteria. The following filter settings were used: EEG: sensitivity $7 \mu \mathrm{V} / \mathrm{mm}$, TC (time constant) $0.3 \mathrm{~s}, \mathrm{HI}$ (high frequency filter) $70 \mathrm{~Hz}$. EOG: sensitivity $30 \mu \mathrm{V} / \mathrm{mm}$, TC $2.0 \mathrm{~s}$, HI $35 \mathrm{~Hz}$; EMG: sensitivity $5 \mu \mathrm{V} / \mathrm{mm}$, TC $0.03 \mathrm{~s}, \mathrm{HI} 500 \mathrm{~Hz}$. Sleep EEG recordings were scored visually by experienced raters according to Rechtschaffen and Kales (1968) criteria. The raters were blind to the experimental conditions.

Sleep recordings were evaluated for parameters of sleep continuity and architecture, and REM sleep. Sleep continuity variables included: (1) sleep efficiency: ratio of total sleep time (TST) to time in bed (TIB) $\times 100 \%$ : (2) sleep onset latency: time from lights out until sleep onset (defined as first epoch of stage 2); (3) number of awakenings: at least one epoch of stage wake during sleep period time (SPT $=$ time from sleep onset till the final awakening during the record).
Sleep architecture variables included: amounts of stages wake, 1, 2, 3, 4, and REM expressed as percentage of SPT. REM sleep variables were: (1) REM latency: time from sleep onset till the first epoch of REM sleep; (2) REM latency corrected was calculated by subtracting wake time between sleep onset and the first REM period from REM latency; (3) duration of the first REM period in min; (4) total number of rapid eye movements during the whole night; (5) eye movement density of the first REM period in \%; (6) total REM density, i.e., eye movement density of all REM periods taken together in \% (REM density is defined as the ratio of $3 \mathrm{~s}$ mini-epochs per REM period including at least one rapid eye movement to the total number of 3-s miniepochs per REM sleep $\times 100 \%$ ).

\section{Serum Tryptophan}

Blood samples for determination of serum tryptophan were obtained at $17.00 \mathrm{~h}$ and at $22.00 \mathrm{~h}$ on days 2 and 4 and at $8.00 \mathrm{~h}$ on days 3 and 5 . Total serum tryptophan was determined by the perchloric acetic acid (PCA) precipitation method (coefficient of variation $8.2 \%$ ).

\section{Psychometric Measurements}

To rule out psychiatric disorders in our subjects, a psychiatric interview as well as a Hamilton depression (HAMD) rating (21-item scale) was obtained by an experienced rater before inclusion into the study. To evaluate behavioral effects of tryptophan depletion, a set of psychometric rating and self-rating scales was completed on days 2 and 4 at $17.00 \mathrm{~h}$ and $22.00 \mathrm{~h}$ and on days 3 and 5 at $7.00 \mathrm{~h}$. Several subjective rating scales were used to determine how the subjects felt at the moment: BF-S (Befindlichkeitsskala, a scale with 28 items predominantly focusing on affective states, $v$. Zerssen [1986]), B-L (Beschwerdeliste, a list with 24 somatic complaints, v. Zerssen [1986]), and analogue scales being divided in six grades for the items mood, anxiety, and tension. In addition, the 6-item version of the HAMD rating (items: depressive mood, feelings of guilt, work, and interest, psychomotor retardation, psychic anxiety, and physical symptoms, Bech at al. 1975) was repeated at the same time points.

\section{Statistical Evaluation}

For descriptive purposes, means and standard deviations (SD) were calculated. The polysomnographic parameters of the tryptophan depletion session and the control session were analyzed by a one-way analysis of variance (ANOVA) for repeated measurements. In addition Student's $t$-tests for dependent samples were calculated to compare the tryptophan depletion and the sham depletion condition. Possible gender effects were 
evaluated by a two-factorial ANOVA (factor treatment and factor gender) and by $t$-tests between the tryptophan depletion and sham condition for the two subgroups. The level of significance was set at $p \leqslant .05$.

\section{RESULTS}

\section{Tryptophan Depletion}

Figure 1 demonstrates that tryptophan serum concentrations did not differ under baseline conditions in both experimental sessions. After the low protein diet, i.e. on day 4 at $17.00 \mathrm{~h}$ tryptophan levels were $17 \%$ decreased compared to baseline on day 2 at $17.00 \mathrm{~h}$. In the subjects who received the tryptophan-substituted diet, serum tryptophan was $20 \%$ increased compared to baseline. At 22.00 h, i.e., 4 hours after the tryptophan-free amino acid drink or the tryptophan-containing drink (sham depletion), respectively, serum tryptophan levels were $85 \%$ decreased and $144 \%$ increased, respectively, compared with baseline values.

\section{Sleep Parameters}

Baseline Nights (Night 2). Mean sleep parameters were almost identical on both baseline nights of the two experimental sessions (Table 1).

Effect of the Diet on Sleep (Night 3). There were no significant changes of sleep parameters after the lowprotein diet compared with baseline sleep parameters. This was true for both the tryptophan-depletion as well as the sham-depletion session. The comparison between the nights after the low protein diet and the tryptophan-substituted diet did not show any significant differences.

Effect of Sham Depletion (Placebo) Compared with Baseline Sleep Parameters. After the control drink, none of the sleep parameters differed significantly compared with baseline.

Effect of Tryptophan Depletion Compared with Baseline Sleep Parameters. After tryptophan depletion, a worsening of nocturnal sleep continuity was observed with a significant increase of the number of waking pe-

\section{Serum-Tryptophan Concentration}

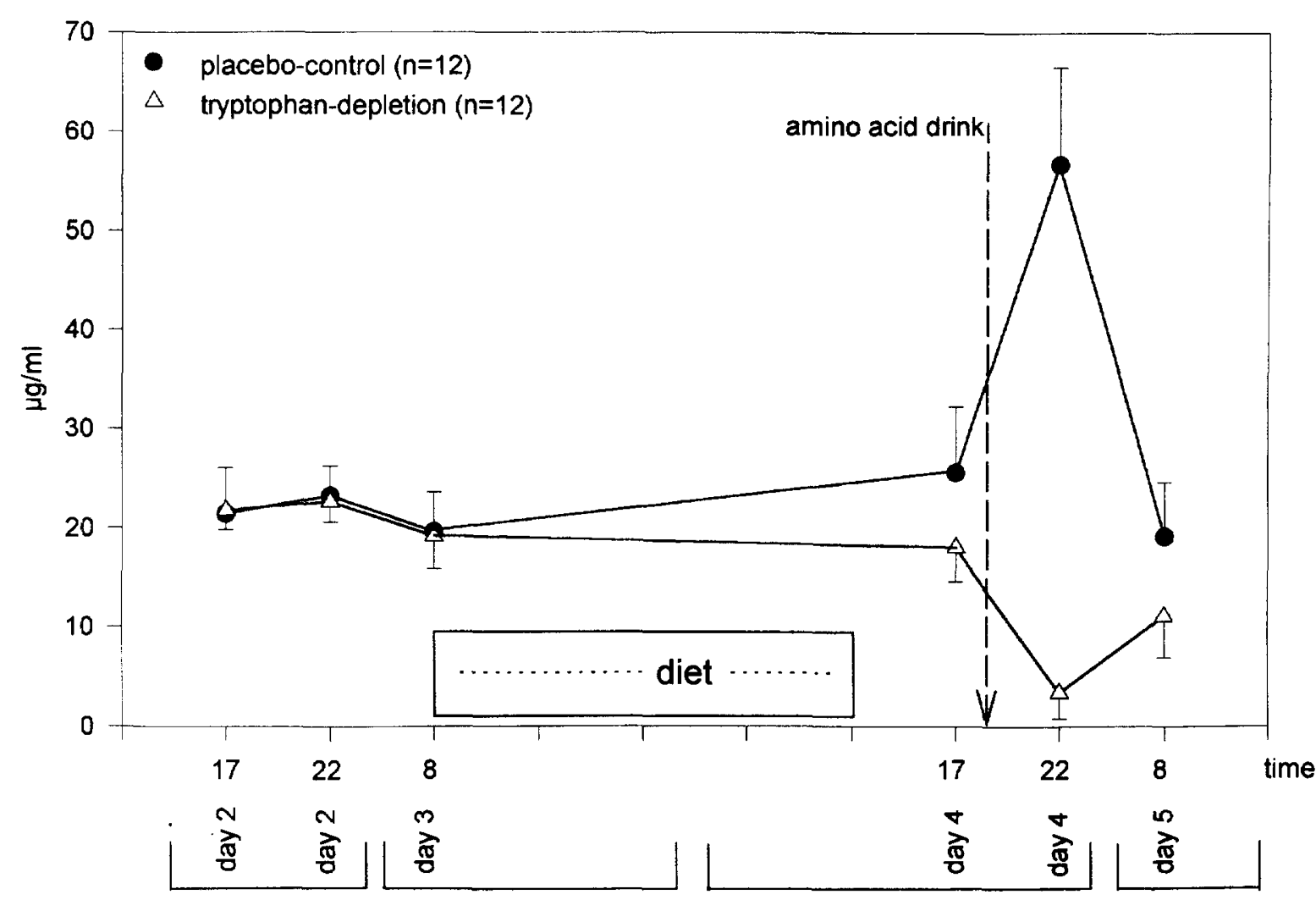

Figure 1. Mean serum tryptophan levels $(x \pm \mathrm{SD}, n=12)$ under baseline conditions on day 2 , and on day 3 at $8.00 \mathrm{~h}$, after the low protein diet on day 4 at $17.00 \mathrm{~h}$ and 4 and $14 \mathrm{~h}$ after a tryptophan-free (tryptophan depletion) or a tryptophan-containing (placebo control) amino acid drink, which was given at $18.00 \mathrm{~h}$ on day 4 . 


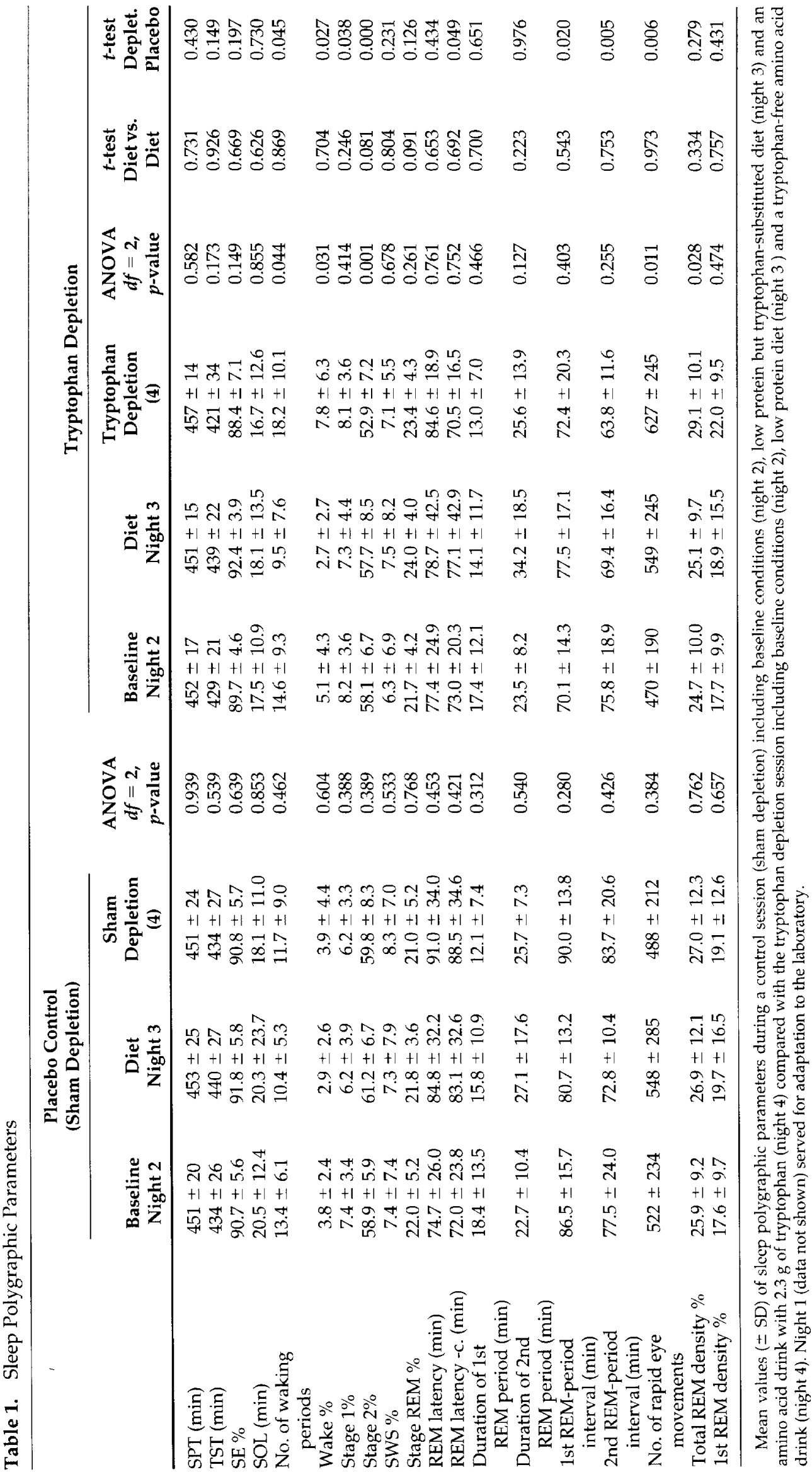




\section{No. of waking periods}

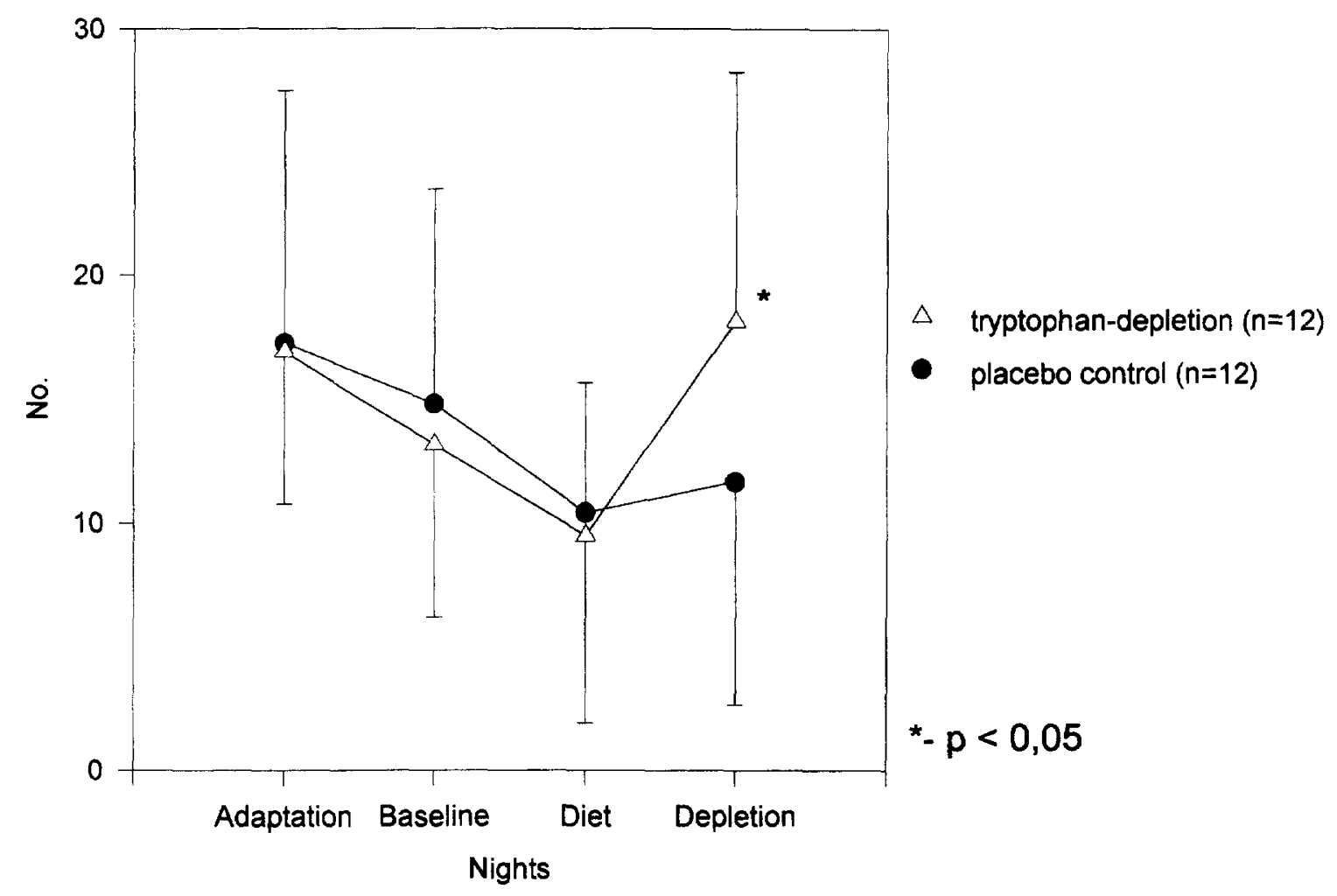

Figure 2. Mean number of waking periods during sleep over 4 consecutive nights in 12 healthy subjects ( \pm SD). Five hours before the fourth night subjects received the tryptophan-free (tryptophan depletion) or tryptophan-containing (placebo control) amino acid mixture.

riods (Figure 2) and the amount of wake time percentage compared with baseline. The overall sleep efficiency, however, was not reduced. The amount of stage 2 sleep was significantly diminished, whereas the amount of slow-wave sleep remained unchanged. REM latency (Figure 3) and also "corrected" REM latency were not significantly altered by tryptophan depletion compared with baseline conditions. However, phasic REM parameters like the total number of eye movements (Figure 4) and the overall REM density increased significantly.

Effect of Tryptophan Depletion Compared with Sham Depletion (Placebo). Several significant differences were found when comparing the effects of tryptophan depletion and those of sham depletion. After the tryptophan-free amino acid drink, the number of waking periods (Figure 2), wake percentage and stage 1 were significantly higher, the amount of stage 2 was significantly lower (Figure 5) than after the tryptophan-containing amino acid drink. The total number of eye movements was markedly and significantly higher after tryptophan depletion (Figure 4), whereas REM den- sity did not differ significantly in both experimental conditions. There were also significant differences with regard to the length of the first and second REM period interval, which were shorter after tryptophan depletion than after sham depletion. The length of the third REM period interval and REM latency (Figure 3 ) did not differ in both paradigms. There was, however, a significant difference of "corrected" REM latency, which was lower after tryptophan depletion compared with sham depletion.

Gender Differences. There were no significant sexspecific effects of the different experimental conditions on sleep EEG measures except for sleep efficiency, which was lowered after tryptophan depletion in the male subjects (tryptophan depletion: $84.9 \pm 7.7 \%$, control session: $91.1 \pm 6.5 \%, p=.02$ ) but not in the female subjects (tryptophan depletion: $91.8 \pm 4.6 \%$, control session: $90.6 \pm 5.3 \%, p=.59$ ).

Psychometric Measurements. No significant differences of mood, anxiety, and other subjective feelings were observed before and during the first 4 hours after 


\section{REM-latency (min)}

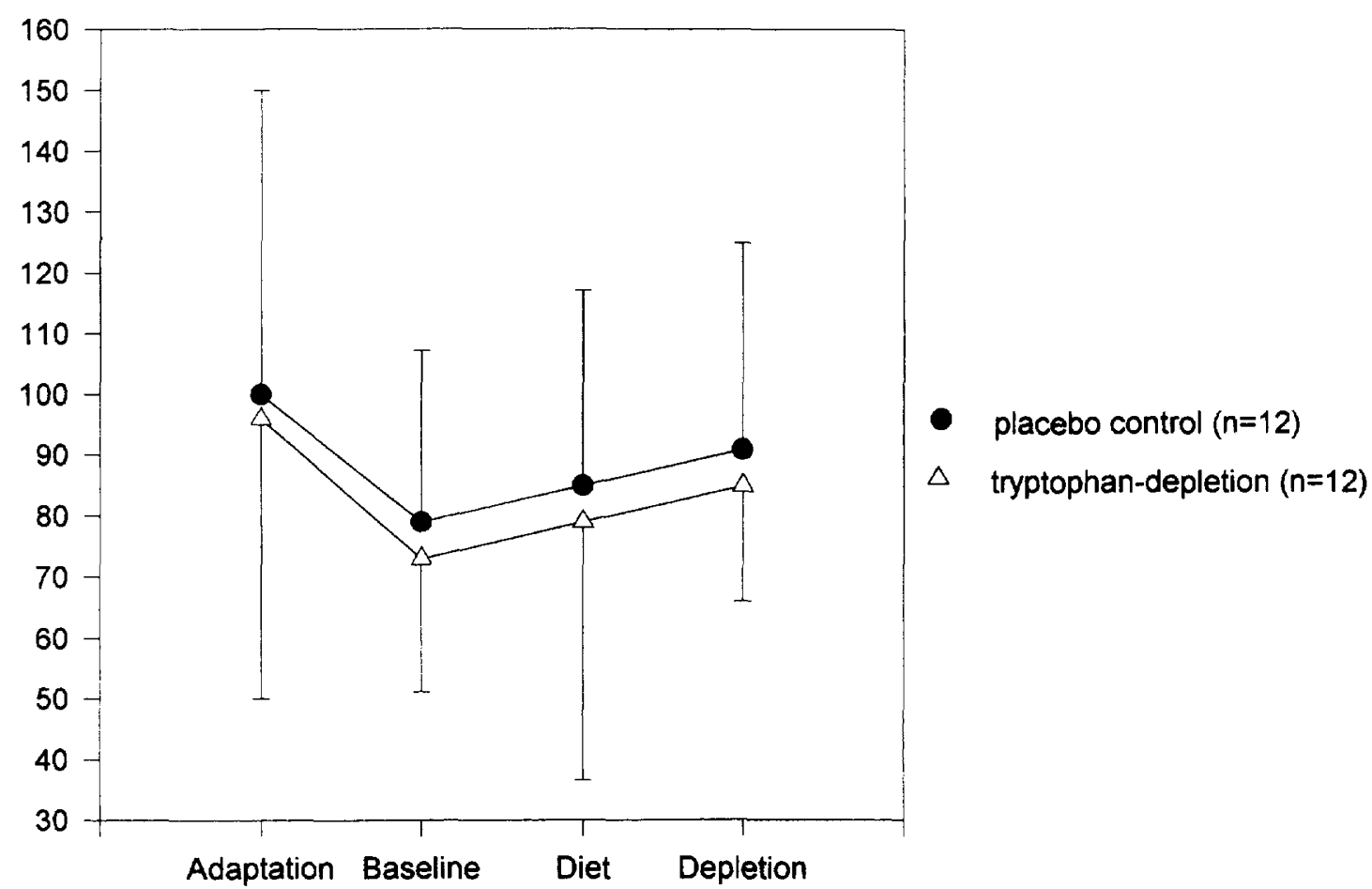

n. s.

Figure 3. Mean REM latency ( $x \pm \mathrm{SD}$ ) during 4 consecutive nights in 12 healthy subjects. Five hours before the fourth night subjects received the tryptophan-free (tryptophan depletion) or tryptophan-containing (placebo control) amino acid mixture.

the tryptophan-free amino acid drink (Table 2) on day 4 compared with sham depletion and compared with baseline conditions on day 2. However, on the morning $(7.00 \mathrm{~h})$ after tryptophan depletion, slightly higher mean values for tension $(7.00 h, p=.04)$ and lower mean values for good mood ( $p=.04)$ were found compared with the morning after sham depletion (Table 2). The mean total score of subjective complaints measured by the B-L scale was also higher on the morning after tryptophan depletion compared with the morning after sham depletion $(p=.03)$. Generally, the changes on all scales were mild. Using the 6-item HAMD-scale, no significant changes were found at any of the time points.

Statistical analysis did not show specific effects due to gender except for the BF-S-score on the morning after tryptophan depletion being significantly higher in males but not in females compared with the morning after sham depletion.

\section{DISCUSSION}

The major finding of this study is a significant effect of an experimentally induced serotonin deficiency by tryptophan depletion on sleep parameters in healthy subjects. This result proves this technique to exhibit significant central nervous system effects in humans. This is in line with the studies reporting central nervous effects in terms of a lowering of mood in remitted depressed patients (Delgado et al. 1990) and in young men at genetic risk for major affective disorders (Benkelfat et al. 1994). However, in addition to these authors, who investigated changes of mood and other psychometric measurements, our study primarily focused on sleep EEG. The methodology of tryptophan depletion was performed according to Delgado et al. (1990) except the time of administration of the amino acid drink, which was administered at $18.00 \mathrm{~h}$ in the evening in our study in contrast to most other authors who gave the drink in the morning. This time of the day was chosen to induce a maximum fall of serum tryptophan immediately before the beginning of sleep recording. In fact, tryptophan levels showed an $85 \%$ decrease at $22.00 \mathrm{~h}$ compared to baseline. This decrease is comparable to studies like those of Delgado et al. (1990), Benkelfat et al. (1994) and Cleare and Bond (1995) who reported an $83 \%, 89 \%$, or $80 \%$ drop, respectively, after the amino acid drink devoid of tryptophan. After sham depletion, 


\section{Total No. of rapid eye movements}

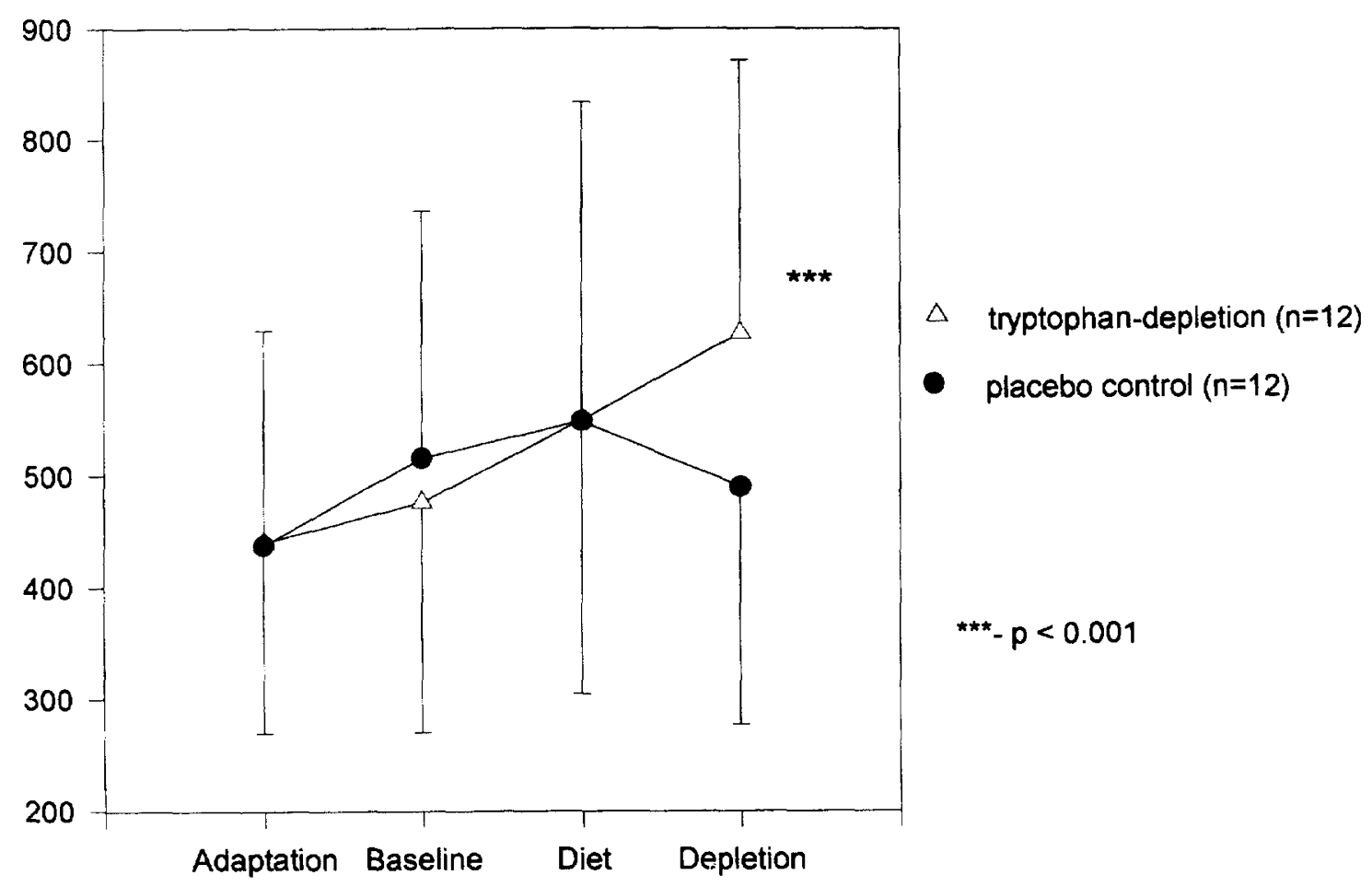

Figure 4. Mean total number of rapid eye movements per night ( $x \pm S D)$ during 4 consecutive nights in 12 healthy subjects. Five hours before the fourth night subjects received the tryptophan-free (tryptophan depletion) or tryptophan-containing (placebo control) amino acid mixture.

i.e., administration of an amino acid drink containing tryptophan, which should represent a placebo control, on the other hand, we observed a $144 \%$ increase of serum tryptophan at $22.00 \mathrm{~h}$ shortly before the start of polysomnographic recordings. The amount of $2.3 \mathrm{~g}$ contained in the amino acid drink that served for "sham" depletion is similar to the amount of tryptophan used for sleeping pills. However, because in the control condition tryptophan was administered together with other large neutral amino acids (LNAAs), which compete with tryptophan at the blood brain barrier, the availability of tryptophan for the brain may not necessarily increase in spite of the $144 \%$ rise of serum tryptophan. Menkes et al. (1994) and Sharma et al. (1997), who measured the ratio between plasma tryptophan and the LNAAs, found slight decreases of this ratio after the control drink, which contained 1.9 and $2.3 \mathrm{~g}$ of tryptophan, respectively. Based on these studies we suppose that in our investigation the control drink did neither enhance nor markedly decrease the tryptophan availability for the central nervous system (CNS). In fact, we did not find any significant effects of the control drink on sleep parameters and on mood compared with baseline. Therefore, the control session can be regarded as a placebo.
Treatment with tryptophan alone in different oral dosages without the other LNAAs resulted in significant effects on human sleep, at least in a part of the studies performed in healthy subjects, insomniacs and psychiatric patients (for review, see Hartmann and Greenwald 1984; Mendelson 1987). Some of these studies reported an increased total sleep time and increased slow-wave sleep after tryptophan, whereas with regard to REM sleep, results were either negative or not consistent among the studies.

The low protein (and low tryptophan) diet alone as well as the tryptophan-substituted diet (placebo condition) had no significant effects on any of the sleep parameters. The low tryptophan diet was used $11 / 2$ days before the amino acid challenge to enhance its tryptophan-depleting effect. Serum levels of tryptophan were not measured at $22.00 \mathrm{~h}$ before the sleep recording, which focused on the effect of the diet alone. One hour before the amino acid challenge, i.e., after about $11 / 2$ days of the low tryptophan diet, tryptophan concentrations were reduced by $17 \%$ compared to a $20 \%$ increase after the tryptophan-substituted diet compared with baseline, respectively. These only minor effects on serum tryptophan levels may explain the lack of significant effects of the diet alone on sleep parameters. 


\section{Sleep stage II in \% of SPT}

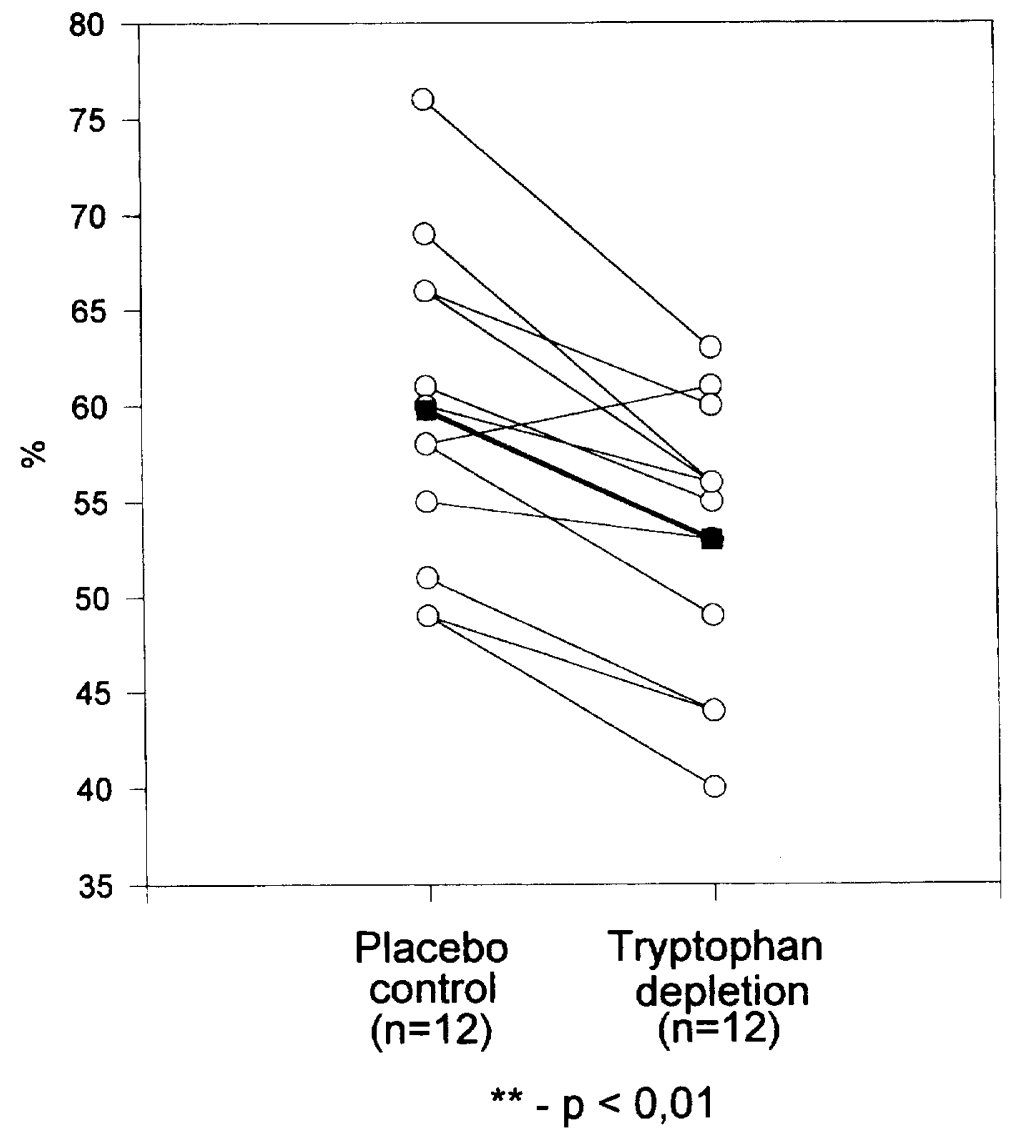

Figure 5. Individual percentages of the amount of stage 2 sleep during night 4 in 12 healthy subjects, who had received either a tryptophan-free (tryptophan depletion) or tryptophan-containing (placebo control) amino acid mixture $5 \mathrm{~h}$ before sleep.
After tryptophan depletion we found significantly more waking periods and more wake percentage and significantly less sleep spent in non-REM stage 2, whereas the amounts of slow-wave sleep and of REM sleep were not significantly changed compared with both baseline conditions and compared with sham depletion. This means that in our study a lowering of brain serotonin levels slightly worsened sleep continuity in normal sleepers. The significant increase in wake time and the marked decrease in non-REM stage 2, which represents the major part of sleep in humans, corresponds well with earlier findings on experimental serotonin deple-

Table 2. Psychometric Measurements Before and After Sham Depletion (Placebo) or Tryptophan Depletion

\begin{tabular}{|c|c|c|c|c|c|c|c|c|}
\hline & \multicolumn{4}{|c|}{$\begin{array}{l}\text { Sham Depletion } \\
\text { (Placebo Control) }\end{array}$} & \multicolumn{4}{|c|}{ Tryptophan Depletion } \\
\hline & $\begin{array}{r}\text { Day } 4 \\
17.00 \mathrm{~h}\end{array}$ & $\begin{array}{c}\text { Day } 4 \\
20.00 \mathrm{~h} \\
\end{array}$ & $\begin{array}{c}\text { Day } 4 \\
22.00 \mathrm{~h}\end{array}$ & $\begin{array}{l}\text { Day } 5 \\
7.00 \mathrm{~h}\end{array}$ & $\begin{array}{r}\text { Day } 4 \\
17.00 \mathrm{~h} \\
\end{array}$ & $\begin{array}{r}\text { Day } 4 \\
20.00 \mathrm{~h}\end{array}$ & $\begin{array}{c}\text { Day } 4 \\
22.00 \mathrm{~h}\end{array}$ & $\begin{array}{l}\text { Day } 5 \\
7.00 \mathrm{~h}\end{array}$ \\
\hline HAMD 6-item & $0.17 \pm 0.39$ & - & $0.42 \pm 0.67$ & $0.33 \pm 0.89$ & $0.83 \pm 1.8$ & - & $0.42 \pm 1.17$ & $0.75 \pm 1.49$ \\
\hline BF-S score & $5.8 \pm 8.5$ & - & $10.6 \pm 12.7$ & $4.8 \pm 4.9$ & $9.9 \pm 9.9$ & - & $9.4 \pm 6.8$ & $9.1 \pm 6.6$ \\
\hline B-L score & $2.5 \pm 2.6$ & - & $5.1 \pm 4.1$ & $1.5 \pm 2.1$ & $4.75 \pm 4.1$ & - & $5.0 \pm 4.8$ & $\begin{aligned} 3.75 & \pm 4.0^{a} \\
p & =0.03\end{aligned}$ \\
\hline Tension & $1.33 \pm 0.65$ & $1.33 \pm 0.65$ & $1.17 \pm 0.39$ & $1.0 \pm 0.0$ & $1.58 \pm 0.67$ & $1.41 \pm 0.51$ & $1.25 \pm 0.45$ & $\begin{aligned} 1.33 & \pm 0.49^{a} \\
p & =0.04\end{aligned}$ \\
\hline Anxiety & $1.0 \pm 0.0$ & $1.25 \pm 0.62$ & $1.08 \pm 0.29$ & $1.0 \pm 0.0$ & $1.17 \pm 0.58$ & $1.08 \pm 0.29$ & $1.0 \pm 0.0$ & $1.0 \pm 0.0$ \\
\hline Good mood & $2.33 \pm 1.44$ & $1.67 \pm 1.15$ & $2.0 \pm 1.12$ & $2.25 \pm 1.29$ & $2.17 \pm 1.34$ & $2.08 \pm 1.67$ & $2.0 \pm 1.3$ & $\begin{aligned} 1.58 & \pm 1.16^{a} \\
p & =0.04\end{aligned}$ \\
\hline
\end{tabular}


tion in animals by either parachlorophenylalanine (PCPA), which inhibits tryptophanhydroxylase or by destruction of the raphe nuclei, which causes profound insomnia in animals (Jouvet 1972). In the view of the monoaminergic/cholinergic regulation of the nonREM-REM cycles, one might assume that a weakening of the serotonergic system would result in an imbalance with a relative preponderance of the cholinergic system. Because it has been shown consistently that cholinergic stimulation induces arousal (Sitaram et al. 1977; Riemann et al. 1994a), an indirect enhancement of the cholinergic tone by tryptophan depletion might explain the significant increase of waking periods and wake percentage.

Statistical analysis did not reveal significant effects on sleep parameters due to gender except for sleep efficiency, which was significantly lowered by tryptophan depletion in the male subjects, but not in the females. Because, however, all other changes of sleep continuity and sleep architecture parameters were not dependent on gender, we conclude from this preliminary result that tryptophan depletion is likely to affect sleep in a sexindependent manner. However, only a further study with a larger sample size can answer this question more clearly.

Our results are not in line with studies by Moja et al. $(1979,1984)$ who reported decreased REM sleep and increased non-REM sleep in animals after a tryptophanfree diet and increases in stage 4 in humans who received a tryptophan-free diet. Lanoir et al. (1981) performed a long-term study up to 16 weeks and found increases in wakefulness and small decreases in non-REM and REM sleep, findings which-apart from the changes in REM sleep-are more similar to our findings in healthy humans.

Another significant finding of this study is an increase of the total number of eye movements compared with baseline and compared with sham depletion and an increased REM density compared with baseline sleep parameters, whereas the total amount of REM sleep was not significantly changed. This indicates a stronger influence of serotonergic pathways on the phasic events of REM sleep such as eye movements compared with the tonic aspects of REM sleep. The increase of rapid eye movements after tryptophan depletion indicates a suppressive effect of serotonin on phasic REM parameters in men. This agrees with a variety of animal studies showing that serotonin depletion by PCPA (Wu and Siegel 1990) or lesions of the serotonin dorsal raphe (Simon et al. 1973) produce a release of ponto-geniculooccipital (PGO) waves, which are closely related to the phasic events of paradoxical sleep such as rapid eye movements (Siegel 1994).

One question of this investigation was that tryptophan depletion would result in similar changes of the sleep EEG as have been described in depressive disorders such as a reduced REM latency (Kupfer 1976), in- crease in REM sleep percentage and increased REM density (for review, see Benca et al. 1992). This was not entirely proven, at least not concerning REM latency and the amount of REM sleep. Several studies demonstrated the strong impact of the cholinergic system on REM latency (Berger et al. 1989; Gillin et al. 1991; Riemann et al. 1994b) and that polysomnographic changes seen in depression may reflect a relative preponderance of the cholinergic as compared to the monoaminergic pathways. A weakening of monoaminergic neurotransmission would result in REM sleep disinhibition as being present in depression. Recently Bhatti et al. (1995a,b) reported an increase of REM sleep and a diminution of REM latency after tryptophan depletion in both normal controls and in remitted depressed patients. The patients were medicated with serotonin reuptake inhibitors and sleep parameters before tryptophan depletion reflect typical polysomnographic changes usually observed after antidepressant treatment such as REM sleep suppression, which was normalized after tryptophan depletion. In this respect, the results obtained in the remitted depressed patients are not directly comparable to ours. However, Bhatti et al. (1995b) also found a significant reduction of REM latency after tryptophan depletion in healthy subjects compared with baseline. These authors, however, do not report data from a placebo control session. In our study, the effect of tryptophan depletion on tonic REM parameters was less pronounced, because REM latency and the amount of REM sleep percentage were not significantly altered. When comparing the effect on REM sleep percentage in both studies, the differences were small, because we found an unsignificant $1.7 \%$ increase of REM sleep and also Bhatti et al. (1995b) reported an unsignificant 3.9\% increase after the same tryptophan-free amino acid drink as in our study. When correcting REM latency for intermittent wake time ("corrected REM latency") we also found a significant reduction after tryptophan depletion compared with the control condition. There were also pronounced effects on the first and second REM period interval, which were significantly decreased by tryptophan depletion. This effect is consistent with the marked reduction of stage 2 non-REM sleep. This shortening of the first and second REM period intervals indicates an even stronger CNS effect of the tryptophan depletion at around $1.00 \mathrm{~h}$ and $3.00 \mathrm{~h}$ compared with the first hours of the sleep period. One might therefore assume that the nadir of the experimentally induced serotonin deficiency occurs about 7 to 9 hours after the amino acid drink. Such a presumed time course of the effects might partly explain the strong effect of tryptophan depletion on REM latency reported by Bhatti et al. (1995a) in remitted depressed patients, because in this study the drink was administered 8 hours and not 5 hours prior to bedtime as in our study and as in Bhatti's and co-workers study (1995b) in healthy subjects. 
With regard to the serotonergic system, obsessive compulsive disorder (OCD) is of special interest because these patients more or less selectively improve under pharmacological treatment with serotonergic antidepressants but-contrary to depressed patients-not with noradrenergic antidepressants (Greist et al. 1995). This and other findings lead to the generation of the serotonin hypothesis of OCD, (Barr et al. 1992) which implies a dysfunctional serotonergic system playing a pathophysiological role in these patients. In a recent analysis of 37 unmedicated OCD patients studied in our sleep laboratory, we found a decreased amount of stage 2 sleep and an increase of phasic REM parameters in OCD patients compared to age- and sex-matched healthy controls, whereas REM latency did not differ between patients and controls (Voderholzer et al. 1996). This striking similarity with our findings after tryptophan depletion in healthy subjects may indirectly support the interpretation that the polysomnographic changes in OCD patients are caused by a disturbance of the serotonergic system.

Barr et al. (1994) and Smeraldi et al. (1996) investigated the effects of tryptophan depletion in OCD patients and did not find a worsening of obsessions and compulsions; however, the first group reported-similar as to the studies in depression-a worsening of mood. If one assumes a serotonergic dysfunction in OCD patients, the lack of effect of tryptophan depletion on these patients could be interpreted as follows: obsessions and compulsions as consequences of a disturbed serotonergic system are not further aggravated after experimental serotonin depletion, as it was similarly shown for actually depressed patients. During the depressed state, tryptophan depletion did not further worsen the depressive symptomatology. It would be of interest to study the effect of tryptophan depletion in OCD patients after successful pharmacological or behavioral therapy.

The evaluation of self-ratings revealed slight but significant behavioral effects after tryptophan depletion compared with the control session. These differences did not occur at the time of maximal changes of serum tryptophan $4 \mathrm{~h}$ after the amino acid drink but on the day after in terms of a higher score for the item "tension" and a lower score for the item "good mood." Generally, no marked or clinically observable changes of mood or behavior occurred, which agrees with Benkelfat et al. (1994) who did not report a significant lowering of mood in healthy subjects without genetic risk for affective disorders. The small self-rated changes in mood on the morning after tryptophan depletion in our study might also be an unspecific effect caused by a slightly worse sleep quality.

In conclusion, our data demonstrated a significant impact of experimental serotonin deficiency on sleep continuity and on the phasic aspects of REM sleep.

\section{ACKNOWLEDGMENT}

The authors thank Dr. G. Huether for determination of serum tryptophan concentrations.

\section{REFERENCES}

Barr L, Goodman W, Price L (1992): Acute exacerbation of body dysmorphic disorder during tryptophan depletion. Am J Psychiatry 149:1406-1407

Barr L, Goodman WK, McDougle CL, Delgado PL, Heninger GR, Charney DS, Price LH (1994): Tryptophan depletion in patients with obsessive-compulsive disorder who respond to serotonin reuptake inhibitors. Arch Gen Psychiatry 51:309-317

Bech P, Gram LF, Dein E, Jacobson O, Vitger J, Bohuys TG (1975): Quantitative ratings of depressive states. Acta Psychiatrica Scand 51:161-170

Benca RM, Obermeyer WH, Thisted RA, Gillin JC (1992): Sleep and psychiatric disorders: A meta-analysis. Arch Gen Psychiatry 49:545-558

Benkelfat C, Ellenbogen M, Dean P, Palmour R, Young S (1994): Mood lowering effect of tryptophan depletion. Arch Gen Psychiatry 51:687-697

Berger M, Riemann D, Hoechli D, Spiegel R (1989): The cholinergic REM sleep induction test with RS 86: State or trait-marker of depression? Arch Gen Psychiatry 46: $421-428$

Berger M, Riemann D (1993): REM sleep in depression-An overview. J Sleep Res 2:211-223

Bhatti T, Gillin JC, Golshan S, Clark C, Demodena A, Schlosser A, Stahl S, Kelsoe J, Rapaport M (1995a): The effect of a tryptophan-free amino acid drink on sleep and mood in normal controls. Sleep Res 24:153

Bhatti T, Gillin JC, Golshan S, Clark C, Demodena A, Schlosser A, Stahl S, Kelsoe J, Rapaport M (1995b): The effect of a tryptophan-free amino acid drink on sleep and mood of patients with major depressive disorder in remission. Sleep Res 24A:267

Cappiello A, Malison RT, McDougle CJ, Vegso SJ, Charney DS, Heninger GR, Price LH (1996): Seasonal variation in neuroendocrine and mood responses to iv L-tryptophan in depressed patients and healthy subjects. Neuropsychopharmacology 15:475-483

Cleare A, Bond A (1995): The effect of tryptophan depletion and enhancement on subjective and behavioural aggression in normal male subjects. Psychopharmacology 118:72-81

Delgado P, Charney D, Price L, Aghajanian G, Landis H, Heninger $G$ (1990): Serotonin and the mechanism of antidepressant action. Arch Gen Psychiatry 47:411-418

Delgado P, Price S, Miller H, Salomon R, Aghajanian G, Heninger F, Charney D (1994): Serotonin and the neurobiology of depression. Effects of tryptophan depletion in drug-free depressed patients. Arch Gen Psychiatry 51:865-874

Ellenbogen MA, Young SN, Dean P, Palmour RM, Benkelfat C (1996): Mood response to acute tryptophan depletion 
in healthy volunteers: Sex differences and temporal stability. Neuropsychopharmacology 15:465-474

Fernstrom JD (1977): Effects of the diet on brain neurotransmitters. Metabolism 26:207-223

Gessa GL, Biggio G, Fadda F, Corsini GV, Tagliamonte A (1974): Effect of oral administration of tryptophan-free amino acid mixtures on serum tryptophan, brain tryptophan and serotonin metabolism. J Neurochem 22: $869-870$

Gillin JC, Sutton FL, Ruiz C, Kelsoe J, Dupont RM, Darko D, Risch C, Golshan S, Janowsky D (1991): The cholinergic rapid eye movement sleep induction test with arecoline in depression. Arch Gen Psychiatry 48:264-270

Greist JH, Jefferson JW, Kobak KA, Katzelnick DJ, Serlin RC (1995): Efficacy and tolerability of serotonin transport inhibitors in obsessive-compulsive disorder. Arch Gen Psychiatry 52:53-60

Hartmann E, Cravens J, List S (1974): Hypnotic effects of tryptophan. Arch Gen Psychiatry 31:394-397

Hartmann E, Greenwald D (1984): Tryptophan and human sleep: An analysis of 43 studies. In Schlossberger $\mathrm{HG}$ Kochen W, Linzen B, Steinhart H (eds), Progress in Tryptophan and Serotonin Research. Berlin, New York, DeGruyter W, pp 297-304

Hill Jr RH, Caudill SP, Philen RM, Bailey SL, Flanders WD, Driskell WJ, Kamb ML, Neeedham LL, Sampson EJ (1993): Contaminants in L-tryptophan associated with eosinophilia myalgia syndrome. Arch Environ Contam Toxicol 25:134-142

Hobson JA, McCarley R, Wyzinsky PW (1975): Sleep cycle oscillation: reciprocal discharge by two brainstem neuronal groups. Science 189:55-58

Jones BE (1994): Basic mechanisms of sleep-wake states. In Kryger MH, Roth T, Dement WC (eds), Principles and Practice of Sleep Medicine. Philadelphia, London, Toronto, Montreal, Sydney, Tokyo, WB Saunders Company, pp 145-162

Jouvet M (1972): The role of monoamine and acetylcholinecontaining neurons in the regulation of the sleep-waking cycle. Ergeb Physiol 64:166-307

Kupfer DJ (1976): REM latency: A psychobiologic marker for primary depressive disease. Biol Psychiatry 11:159-174

Kupfer DJ, Ehlers CL (1989): Two roads to rapid eye movement latency. Arch Gen Psychiatry 46:945-948

Lanoir J, Ternaux JP, Pons C, Lagarde JM (1981): Long-term effects of a tryptophan-free diet of serotonin metabolism and sleep-waking balance in rats. Exp Brain Res 41: 346-357

Meltzer HY, Lowy MT (1987): The serotonin hypothesis of depression. In Meltzer HY (ed), Psychopharmacology: The Third Generation of Progress. New York, Raven Press, pp 513-526

Mendelson WB (1987): Human sleep. Research and Clinical Care. New York London, Plenum Medical Book Company, pp 34-40

Menkes DB, Coates DC, Fawcett JP (1994): Acute tryptophan depletion aggravates premenstrual syndrome. J Affective Disord 32:37-44

Moja EA, Mendelson WB, Stoff DM, Gillin JC, Wyatt RJ
(1979): Reduction of REM sleep by a tryptophan-free amino acid diet. Life Sci 24:1467-1470

Moja EA, Antinoro E, Cesa-Bianchi M, Gessa GL (1984): Increase in stage 4 sleep after ingestion of a tryptophan free diet in humans. Pharmacol Res Commun 16:909-914

Moja EA, Cipolla P, Castoldi D, Tofanetti O (1989): Doseresponse decrease in plasma tryptophan and in brain tryptophan and serotonin after tryptophan-free amino acid mixtures in rats. Life Sci 44:971-976

Neumeister A, Praschak-Rieder N, Heßelmann B, Rao M-L, Glück J, Kasper S (1997): Effects of tryptophan depletion on drug-free patients with seasonal affective disorder during a stable response to bright light therapy. Arch Gen Psychiatry 54:133-138

Nicholson AN, Pascoe PA (1988): Studies on the modulation of the sleep-wakefulness continuum in man by fluoxetine, a 5-HT uptake inhibitor. Neuropharmacology 27: $597-602$

Rechtschaffen A, Kales A (eds) (1968): A Manual of Standardized Terminology, Techniques and Scoring System for Sleep Stages of Human Subjects. Department of Health, Education, and Welfare, Washington DC

Riemann D, Berger M (1990): The effects of total sleep deprivation and subsequent treatment with clomipramine on depressive symptomatology and sleep electroencephalography in patients with a major depressive disorder. Acta Psychiatr Scand 81:24-31

Riemann D, Gann H, Dressing H, Müller WE, Aldenhoff JB (1994a): Influence of the cholinesterase inhibitor galanthamine hydrobromide on normal sleep. Psychiatry Res 51:253-267

Riemann D, Hohagen F, Krieger S, Gann H, Müller WE, Olbrich R, Wark H-J, Bohus M, Löw H, Berger M (1994b): Cholinergic REM induction test: Muscarinic supersensitivity underlies polysomnographic findings in both depression and schizophrenia. J Psychiatr Res 28:195-210

Schneider-Helmert D, Spinweber CL (1986): Evaluation of L-tryptophan for treatment of insomnia: A review. Psychopharmacology 89:1-7

Sharma RP, Shapiro LE, Kamath SK, Soll EA, Watanabe MD, Davis JM (1997): Acute dietary tryptophan depletion: Effects on schizophrenic positive and negative symptoms. Neuropsychobiology 35:5-10

Siegel JM (1994) Brainstem mechanisms generating REM sleep. In Kryger MH, Roth T, Dement WC (eds), Principles and Practice of Sleep Medicine. Philadelphia, London, Toronto, Montreal, Sydney, Tokyo, WB Saunders Company, pp 125-144

Simon RP, Gershon MD, Brooks DC (1973): The role of the raphe nuclei in the regulation of ponto-geniculo-occipital wave activity. Brain Res 58:313-330

Sitaram N, Mendelson WB, Wyatt RJ, Gillin JC (1977): The time-dependent induction of REM sleep and arousal by physostigmine infusion during normal human sleep. Brain Res 122:562-567

Smeraldi E, Diaferia G, Erzegovesi S, Lucca A, Bellodi L, Moja E (1996): Tryptophan depletion in obsessive-compulsive patients. Biol Psychiatry 40:398-402

Voderholzer U, Riemann D, Backhaus J, König A, Böhme R, 
Kiemen A, Berger M, Hohagen F (1996): Polysomnographic studies in patients with obsessive-compulsive disorder. J Sleep Res 5, suppl 1, 246

Wu MF, Siegel JM (1990): Facilitation of the acoustic startle reflex by ponto-geniculo-occipital waves: Effects of PCPA. Brain Res 532:237-241

Yamaoka KA, Myasaka N, Inuo G, Saito I, Kolb JP, Fujita K, Kashiwazaki S (1994): 1,1'-ethylidenebis (tryptophan) (Peak E) induces functional activation of human eosinophils and interleukin 5 production from $\mathrm{T}$ lymphocytes:
Association of eosinophilia-myalgia syndrome with a L-tryptophan contaminant. J Clin Immunol 14:50-60

Young SN, Smith SE, Pihl RO, Ervin FR (1985): Tryptophan depletion causes a rapid lowering of mood in normal males. Psychopharmacology 87:173-177

Zerssen von D (1986): Clinical Self-Rating Scales (CSRS) of the Munich Psychiatry Information System (PSYCHIS München). In Sartorius N, Ban TA (eds), Assessment of Depression. Berlin, Heidelberg, New York, Tokyo, Springer, pp 270-303 